\title{
Living with Narcolepsy: Current Management Strategies, Future Prospects, and Overlooked Real-Life Concerns
}

This article was published in the following Dove Press journal:

Nature and Science of Sleep

\author{
Emily C Barker' \\ Julie Flygare ${ }^{2}$ \\ Shalini Paruthi ${ }^{3,4}$ \\ Katherine M Sharkey $\mathbb{D}^{5-7}$ \\ 'Case Western Reserve University \\ School of Medicine, Department of \\ Pediatrics, Cleveland, OH, USA; ${ }^{2}$ Project \\ Sleep, Los Angeles, CA, USA; ${ }^{3}$ Sleep \\ Medicine and Research Center, St. Luke's \\ Hospital, St. Louis, MO, USA; ${ }^{4}$ Saint Louis \\ University School of Medicine, \\ Departments of Medicine and Pediatrics, \\ St. Louis, MO, USA; ${ }^{5}$ Department of \\ Medicine, The Warren Alpert Medical \\ School of Brown University, Providence, \\ RI, USA; 'Department of Psychiatry \& \\ Human Behavior, The Warren Alpert \\ Medical School of Brown University, \\ Providence, RI, USA; ${ }^{7}$ Rhode Island \\ Hospital, Providence, RI, USA
}

Correspondence : Katherine M Sharkey Alpert Medical School, Brown University, 233 Richmond Street, Providence, RI 02906 Tel + I 40I-863-2450

$\mathrm{Fax}+$ I 40I-863-2940

Email Katherine_Sharkey@brown.edu

\begin{abstract}
Narcolepsy is a neurological disorder of the sleep-wake cycle characterized by excessive daytime sleepiness (EDS), cataplexy, nighttime sleep disturbances, and REM-sleep -related phenomena (sleep paralysis, hallucinations) that intrude into wakefulness. Dysfunction of the hypocretin/orexin system has been implicated as the underlying cause of narcolepsy with cataplexy. In most people with narcolepsy, symptom onset occurs between the ages of 10 and 35 years, but because the disorder is underrecognized and testing is complex, delays in diagnosis and treatment are common. Narcolepsy is treated with a combination of lifestyle modifications and medications that promote wakefulness and suppress cataplexy. Treatments are often effective in improving daytime functioning for individuals with narcolepsy, but side effects and/or lack of efficacy can result in suboptimal management of symptoms and, in many cases, significant residual impairment. Additionally, the psychosocial ramifications of narcolepsy are often neglected. Recently two new pharmacologic treatment options, solriamfetol and pitolisant, have been approved for adults, and the indication for sodium oxybate in narcolepsy has been expanded to include children. In recent years, there has been an uptick in patient-centered research, and promising new diagnostic and therapeutic options are in development. This paper summarizes current and prospective pharmacological therapies for treating both EDS and cataplexy, discusses concerns specific to children and reproductive-age women with narcolepsy, and reviews the negative impact of health-related stigma and efforts to address narcolepsy stigma.
\end{abstract}

Keywords: narcolepsy, cataplexy, sleepiness, hypersomnia, solriamfetol, pitolisant, sodium oxybate, children, pregnancy, lactation, stigma

\section{Introduction}

Narcolepsy is a chronic neurologic disorder that affects the stability of sleep and wakefulness. ${ }^{1}$ All individuals with narcolepsy have excessive daytime sleepiness (EDS), and most experience sleep paralysis, hypnogogic and/or hypnopompic hallucinations, and/or disrupted sleep at night. ${ }^{2}$ Cataplexy, a phenomenon of sudden, brief loss of muscle tone during wakefulness, is another defining feature of narcolepsy. ${ }^{1}$ Additionally, many people with narcolepsy report difficulties with concentration, cognition, and daytime fatigue. ${ }^{3}$

Diagnosis of narcolepsy requires confirmation of EDS with a multiple sleep latency test (MSLT) — defined as an average sleep latency of $<8$ minutes during 5 sleep opportunities across the day-as well as two or more REM-onset naps. ${ }^{4}$ Because of converging evidence that narcolepsy with cataplexy results from 
dysfunction in the neural hypocretin/orexin pathways that contribute to sleep-wake regulation, ${ }^{5}$ low hypocretin-1 levels $(\leq 110 \mathrm{pg} / \mathrm{mL})$ measured in cerebrospinal fluid (CSF) can also be used to diagnose narcolepsy in centers where the test is available. At the time of publication, clinical testing for Orexin-A/Hypocretin-1 in Spinal Fluid is available through Mayo Clinic Laboratories. ${ }^{6}$ Narcolepsy is categorized into two types based on clinical presentation and associated underlying biomarkers. Narcolepsy Type 1 (NT1) is defined by the presence of cataplexy and is nearly always associated with low hypocretin levels and/or impaired hypocretin signaling, whereas Narcolepsy Type 2 (NT2) is diagnosed when cataplexy does not occur and hypocretin deficiency/dysfunction is not observed. Partial deficiency of hypocretin can exist in the absence of cataplexy, however, indicating that some cases of NT2 may be caused by less-extensive impairment to hypocretin signaling. ${ }^{7}$ Moreover, the scant neuropathology data in NT2 suggest that although hypocretin neuronal dysfunction is implicated in both types of narcolepsy, hypocretin neuron damage is more circumscribed in NT2 than in NT1. ${ }^{7}$ NT2 is a challenge to diagnose due to non-specificity of symptoms, limitations of diagnostic tests and absence of useful biomarkers, and much remains to be understood about the pathophysiology of this hypersomnia subtype. ${ }^{8}$ Whether NT2 is an entity distinguishable from idiopathic hypersomnia (IH) is also a topic of intense interest, and although this continues to be debated, some differentiating features have been advanced, eg, more disrupted nocturnal sleep and better restorative response to naps in NT2 than $\mathrm{IH}^{8,10}$

Although the exact causes of narcolepsy are still under investigation, evidence points to an immune process that results in damage and/or dysfunction of the hypocretinproducing neurons in the lateral-posterior hypothalamus as a plausible etiology for NT1. ${ }^{5}$ Observations that support immune-mediated NT1 pathophysiology include the association of NT1 development with major histocompatibility complex genes, particularly the HLA-DQB $1 * 06: 02$ allele, ${ }^{11}$ influenza $\mathrm{A}$ and streptococcal infections, and the Pandemrix H1N1 vaccine. ${ }^{12}$ Other areas of active investigation into the causes of narcolepsy include examination of the histamine system, search for other immune mediators, and studies of "secondary narcolepsy" attributed to hypothalamic injury from traumatic, infectious, or demyelinating processes.

Prevalence estimates indicate that narcolepsy affects between 25 and 50 people per $100,000,{ }^{13}$ with higher rates observed in Japanese ${ }^{14}$ and African American ${ }^{15}$ cohorts and lower rates in Israel. ${ }^{16}$ A 2019 study of an insurance database comprised of $>18$ million people found a prevalence rate of 79.4 per 100,000 persons and an incidence rate between 4.87 and 7.67 per 100,000 persons per year. ${ }^{17}$ Variation in prevalence estimates is attributed to differences in case definition across studies and to differences in genetic susceptibility to narcolepsy among different subgroups. ${ }^{13}$ Historically, narcolepsy had been reported to be more common in men than in women, ${ }^{18}$ though more recent epidemiologic evidence shows equal prevalence of narcolepsy between genders or higher prevalence in women. ${ }^{17,19,20}$ Women with narcolepsy report earlier symptom onset than men, and fall asleep more quickly on the MSLT. $^{20}$ Furthermore, in the Doberman Pinscher canine narcolepsy model, cataplexy was at least twice as severe in females as in males. ${ }^{21}$

\section{Diagnostic and Treatment Challenges}

The first symptoms of narcolepsy typically occur between the ages of 10-35 years, though cases of earlier and later presentations have been described. A major challenge is the time from symptom presentation to diagnosis. Studies examining the duration from the first signs of NT1 to diagnosis show a range of 8.4 to 22.1 years with an average delay of $\sim 15$ years. ${ }^{22}$ It is estimated that up to $50 \%$ of people with narcolepsy are undiagnosed. ${ }^{2}$ One study of 52 people with NT1 found the average time gap between symptom onset and medical consultation was $3.2 \pm 5.1$ years and the time from medical presentation to diagnosis was $8.9 \pm 11.0$ years. ${ }^{23}$ It is encouraging, however, that delays appear to be decreasing over the last 5 decades. ${ }^{22,23}$ Although no gender difference in the age of narcolepsy symptom onset has been observed, women may experience a delay in diagnosis compared to men; one study showed a duration of 16 years from the onset of symptoms to diagnosis in men and 28 years in women. ${ }^{20}$

Even when the narcolepsy is suspected, diagnosis can be elusive. Short sleep latencies and sleep onset REM periods can be seen in conditions other than narcolepsy, ${ }^{24,25}$ and although test-retest reliability is high in NT1, more variable MSLT results are observed among individuals with NT2 and idiopathic hypersomnia. ${ }^{26}$ A short REM latency ( $\leq 15$ minutes) during nocturnal polysomnography (NPSG) has high specificity for NT1 
and can aid in diagnosis, ${ }^{27,28}$ though short REM latency on NPSG can also be observed in people with other untreated sleep disorders, night-shift workers, and individuals with insufficient sleep. ${ }^{29}$ From a practical standpoint, many individuals with excessive sleepiness take medications that interfere with multiple sleep latency testing, and tapering and withholding these medications in preparation for the MSLT can cause safety concerns and decrements to quality of life. Measurement of a CSF hypocretin-1 level is a definitive test for NT1, ${ }^{30}$ but challenges remain, such as access and acceptability among individuals with possible narcolepsy. Innovative testing methods using neuroimaging techniques such as positron emission tomography (PET), single-photon emission computed tomography (SPECT), functional magnetic resonance imaging (fMRI), and magnetic resonance spectroscopy (MRS) are being investigated as possible tools for improving diagnosis of narcolepsy. ${ }^{31}$

People with narcolepsy have a higher incidence of certain comorbid conditions, including obesity, depression, anxiety, and other sleep disorders. For instance, a community-based study that compared 68 individuals with narcolepsy to age- and sex-matched controls at diagnosis and after an average follow-up period of 9.9 years found higher rates of obesity, psychiatric conditions, obstructive sleep apnea, endocrine disorders, and low back pain among those with narcolepsy. ${ }^{19}$ A larger study of insurance-coding data that included 9312 people with narcolepsy, found excess rates of anxiety disorders, diabetes, headaches, depression, obesity, periodic limb movement disorder, obstructive sleep apnea, REM behavior disorder, and restless legs syndrome. ${ }^{32}$ Won et $\mathrm{al}^{20}$ examined gender differences in comorbid conditions among people with narcolepsy and found that men were significantly more likely to have a diagnosis of attention-deficithyperactivity disorder (ADHD), whereas women were more likely to be diagnosed with a neurologic or autoimmune disorder.

A limited number of studies have examined the relationship between age and the core symptoms of narcolepsy. Findings suggest that symptoms are dynamic over time and that EDS, cataplexy, and night-time sleep quality may improve with age. ${ }^{33,36}$ Age-related increase in MSLT sleep latency and decrease in sleep onset REM periods (SOREMPs) have been observed in NT1. ${ }^{34}$ Cataplexy has been observed to be significantly less frequent in individuals with NT1 age 65 or older, compared to those younger than 65 years. $^{33}$ Additionally, a 10-year longitudinal study reported improvement and even resolution of cataplexy, along with improved ESS and night-time sleep quality in a large proportion of individuals with NT1. ${ }^{36}$ Another longitudinal study found that in a majority of individuals with NT1, EDS changed little over the 5 years of observation; nevertheless, spontaneous improvement in EDS was observed in $15 \%$ of the cohort, and was tied to milder hypocretin deficiency and more consolidated night-time sleep. ${ }^{35}$ These studies challenge prior assumptions that narcolepsy symptoms are stable and unremitting over time.

Individuals with narcolepsy have higher rates of accidental injury than matched controls, including burns, ${ }^{37}$ bone fractures $^{38}$ and automobile crashes. ${ }^{39}$ In a study that compared car crashes between 282 individuals with NT1, NT2, or idiopathic hypersomnia (IH) and 470 controls, $22.7 \%$ of people with a hypersomnolence condition reported an auto accident in the previous 5 years compared with $14 \%$ of controls. ${ }^{39}$ Crashes were predicted by higher levels of subjective sleepiness, use of caffeinated energy drinks, and reports of daytime napping. Interestingly, those with narcolepsy or IH who had been treated for 5 years or more did not have higher rates of automobile accidents than controls. In the European Union, national restrictions regulate that fitness to drive is assessed prior to issuance or renewal of driving licenses for individuals with certain sleep disorders, including NT1 and NT2. ${ }^{40}$ In the US, most state motor vehicle bureaus expect driving license applicants to selfreport conditions that may compromise fitness to drive, but do not make reporting of medical conditions mandatory and may or may not require further documentation if a condition is self-reported. Only a handful of states require mandatory reporting of a medical condition, including narcolepsy, that may impair driving, as well as documented assessment of fitness to drive. ${ }^{41}$

\section{Treatment Landscape for Narcolepsy}

In 2013, the FDA hosted a patient-focused drug development initiative (PFDDI) for narcolepsy to gather perspectives from individuals living with this condition. Some of the reported therapeutic challenges for the narcolepsy community included variable responsiveness and limited access to available treatment options, as well as intolerable drug side effects. ${ }^{42}$ The Maski et $\mathrm{al}^{43}$ report of the Unite Narcolepsy survey, administered in conjunction with the PFDDI meeting, indicated that $57.1 \%$ of people with narcolepsy experienced daytime sleepiness or fatigue three or more times per day in spite of treatment while only $3.9 \%$ 
reported no daytime sleepiness in response to treatment. The lack of perceived efficacy, as well as side effects, drug interactions, and high costs, likely contribute to inconsistent medication use. A study of 116 people with narcolepsy found that while $55.2 \%$ of study participants took their medications at least $80 \%$ of the time, $12.9 \%$ took their medications only $50-80 \%$ of the time and $31.9 \%$ took their prescribed medications less than $50 \%$ of the time. $^{44}$

It is striking that until recently, only 5 medications had been indicated for the treatment of narcolepsy in the US, reflecting an ongoing need for increased treatment options to combat EDS and cataplexy in narcolepsy. Increased understanding of narcolepsy pathophysiology is enabling exploration of other candidate molecules and in 2019 two new compounds, pitolisant and solriamfetol, were approved in the US for treatment of excessive daytime sleepiness in narcolepsy. Alternative formulations of current treatments aim to increase convenience and adherence to regimens and extended indications are expanding options for the pediatric population. Additionally, prospective treatments, such as orexin agonists, are showing promise in clinical trials and may soon find their way into the armamentarium of treatment options. Nonpharmacological therapies are likely to be effective adjunctive treatment for narcolepsy.

\section{Historic and Current Pharmacologic Treatments}

Historically, stimulants, antidepressants and hypnosedatives have been used to treat EDS and cataplexy associated with narcolepsy. The first compound to demonstrate significant improvement for excessive sleepiness in narcolepsy was amphetamine in 1935 and it is still prescribed today and indicated for that purpose. ${ }^{45,46}$ Methylphenidate has been used to treat EDS in narcolepsy since the 1950s and is considered to have a more favorable safety profile than amphetamine. Methylphenidate and amphetamine are recommended as second- and third-line therapeutics for EDS. They are mechanistically similar, promoting wakefulness by increasing presynaptic dopamine release. ${ }^{45}$

Tricyclic antidepressants (TCAs), including imipramine, clomipramine, desipramine and protriptyline were reported to improve cataplexy symptoms as early as 1960 . They are included as suggested treatments for cataplexy by the American Academy of Sleep Medicine (AASM) and European Federation of Neurological Societies, although rigorous randomized, placebo-controlled clinical trials have not been performed. ${ }^{46,47}$ Intolerable side effects with TCAs are common, as well as rebound cataplexy upon withdrawal. ${ }^{45}$ Although no antidepressants are FDAapproved for the treatment of cataplexy, classes other than TCAs have shown efficacy in reducing cataplexy, and are often better tolerated. Venlafaxine, a selective serotonin and norepinephrine reuptake inhibitor (SNRI), is a firstline recommended therapeutic for cataplexy and shows fewer side effects but has similar withdrawal concerns as TCAs. Fluoxetine, a selective serotonin reuptake inhibitor (SSRI), and selegiline, a monoamine oxidase type $\mathrm{B}$ inhibitor, are also common antidepressant-class recommended treatments for cataplexy. ${ }^{45,47}$

Currently, sodium oxybate, the sodium salt of gamma hydroxybutyrate, is the only medication indicated for both EDS and cataplexy. It is recommended as the first-line therapy for both of these indications, based upon rigorous randomized, placebo-controlled clinical trials, and its indication was recently expanded to include treatment of children $(\geq 7$ years of age) with narcolepsy after a placebo-controlled, double-blind, randomized clinical trial demonstrated the efficacy, safety, and tolerability of sodium oxybate in children and adolescents with narcolepsy. ${ }^{48}$ For individuals who cannot take sodium oxybate, first-line recommended wakepromoting agents are modafinil and armodafinil, in part because their potential for abuse and dependency are lower than amphetamine. Modafinil has been shown to control EDS in narcolepsy at a comparable level to sodium oxybate, but is ineffective in reducing cataplexy, compared to placebo. $^{49}$

\section{New and Prospective Pharmacologic Treatments \\ Solriamfetol}

Solriamfetol, a derivative of phenylalanine, is a dopamine and norepinephrine reuptake inhibitor recently approved in the US for the treatment of EDS in adults with narcolepsy or obstructive sleep apnea. In Phase 2 and 3 clinical trials, solrimfetol was shown to significantly improve wakefulness and reduce sleepiness in these patient populations compared to placebo as shown by Maintenance of Wakefulness Test (MWT) sleep latency and by Epworth Sleepiness Scale (ESS) scores. ${ }^{50,53}$ Solriamfetol inhibits reuptake of the monoamines via interaction at both the dopamine transporter and the norepinephrine transporter and thus by a mechanism distinct from that of the wake-promoting agent modafinil, 
which is thought to bind primarily at the dopamine transporter to inhibit dopamine reuptake. ${ }^{54}$ Solriamfetol also differs mechanistically from the amphetamines as it does not promote the release of norepinephrine. ${ }^{51,55,56}$ These distinctions may account for differences in therapeutic effects between solriamfetol and other wake-promoting drugs. ${ }^{56,57}$ Unlike modafinil, armodafinil and pitolisant (see below), solriamfetol does not interfere with the efficacy of oral contraceptive pills, ${ }^{58}$ potentially offering a more convenient option of nonamphetamine wake-promoting agent to women with narcolepsy passing through their child-bearing years. Solriamfetol treatment also shows no evidence of drug-induced rash, a potential side effect of the wake-promoting agents modafinil and armodafinil. ${ }^{59}$

\section{Pitolisant}

Pitolisant is an antagonist/inverse agonist of the histamine $\mathrm{H}_{3}$ receptor that was approved for the treatment of narcolepsy with or without cataplexy in the European Union in $2016^{60}$ and in the US for the treatment of EDS in adults in August 2019. ${ }^{58}$ The histaminergic system in the central nervous system modulates processes including wakefulness, feeding, and learning and memory consolidation via histamine receptors, $\mathrm{H}_{1}, \mathrm{H}_{2}, \mathrm{H}_{3}$ and $\mathrm{H}_{4}{ }^{61}$ Blocking histamine synthesis, or histamine receptor $\mathrm{H}_{1}$, has been shown to increase cortical slow waves and enhance sleep. Conversely, enhancing the neurotransmission of histamine has been shown to promote wakefulness. The histamine $\mathrm{H}_{3}$ receptor controls the release, synthesis and turnover of histamine and the neuronal activity of histaminergic cells. $^{62}$ Thus, pitolisant acts to promote wakefulness by blocking $\mathrm{H}_{3}$ receptors, which, in turn, increases synaptic histamine release via $\mathrm{H}_{1}$ receptors. ${ }^{63}$

Randomized and open-label clinical trials have demonstrated efficacy of pitolisant in decreasing ESS scores and increasing MWT scores compared to placebo in people with narcolepsy with or without cataplexy, as well as in decreasing severity of cataplexy compared to placebo in people with NT1 ${ }^{64,66}$ These studies also demonstrated that pitolisant is noninferior to modafinil in reducing EDS in people with narcolepsy with or without cataplexy.

\section{Alternative Sodium Oxybate Formulations}

Multiple investigations are underway to identify alternative formulations of sodium oxybate to increase the options with this standard therapy for both EDS and cataplexy. $^{47,58}$ To address the twice-nightly dosing of sodium oxybate, which some individuals find difficult to time correctly or to be an inconvenience, a once-nightly dosing regimen of an extended-release sodium oxybate formulation that uses micropump technology (FT218) is being tested for safety and efficacy. FT218 recently completed Phase III clinical development for treatment of EDS associated with narcolepsy and cataplexy, evaluating safety and efficacy of daily doses of 4.5, 6.0, 7.5 and 9.0 g sodium oxybate compared to placebo. Outcomes include MWT sleep latency, Clinical Global Impression of Change (CGI-C) and mean number of cataplexy attacks. If approved, FT-218 will be the first once-nightly treatment to address both EDS and cataplexy in narcolepsy. ${ }^{67}$

A low-sodium alternative to sodium oxybate, JPZ-258, is a recently developed mixed salts oxybate oral solution. Sodium load for the maximum therapeutic dose of sodium oxybate $(9.0 \mathrm{~g})$ is equivalent to $1640 \mathrm{mg}$ sodium intake, ${ }^{68}$ $71 \%$ of the $2300 \mathrm{mg}$ maximum recommended sodium intake by the American Heart Association, and exceeding the $1500 \mathrm{mg}$ recommended for individuals with conditions associated with sodium sensitivities. ${ }^{69}$ The new formulation, JPZ-258, would reduce sodium intake by up to $92 \%$ and may thus present a safer alternative for people with narcolepsy who also have heart failure, hypertension or renal impairment or for those carrying risk factors for such conditions. Recently completed Phase 3 clinical trials evaluated safety and efficacy in people with narcolepsy with cataplexy and demonstrated significant differences in weekly number of cataplexy attacks as well as improved ESS scores compared to placebo. ${ }^{70,71}$ The US Food and Drug Administration accepted a New Drug Application for JZP-258 for the treatment of cataplexy and EDS in people with narcolepsy ages 7 years and older in March 2020 and it is anticipated it will be commercially available in 2020 .

\section{Hypocretin/Orexin-Based Therapies}

Because the pathophysiology of narcolepsy involves deficiency of the neuropeptide hypocretin- $1,{ }^{72}$ multiple modes of hypocretin ligand-replacement strategies have been pursued as potential therapies for narcolepsy. ${ }^{47}$ Penetration of the blood-brain barrier has proven the greatest challenge in hypocretin-replacement therapy. Considering significant barriers to hypocretin-replacement approaches, nonpeptide small molecule hypocretin receptor agonists represent another avenue for targeting hypocretin deficiency in narcolepsy. TAK-925 is a hypocretin/orexin 2 receptorselective agonist that is under development and has been shown to promote wakefulness in mice. ${ }^{73}$ Phase 1 studies indicate that intravenous TAK-925 is well tolerated in 
people with narcolepsy type 1 and increases wakefulness compared to placebo as measured by MWT and Karolinska Sleepiness Scale. ${ }^{74,75}$ An oral hypocretin/ orexin 2 receptor-selective agonist TAK-994 has also shown increased wakefulness and decreased cataplexy in mouse models and is progressing in phase 1 and phase 2 clinical trials. ${ }^{76,78}$ These strides suggest a promising future for hypocretin/orexin receptor agonism in the treatment of narcolepsy with cataplexy.

\section{AXS-I 2 (Reboxetine)}

Reboxetine is a selective norepinephrine reuptake inhibitor approved for treatment of depression in 40 countries outside of the U.S. ${ }^{58}$ A formulation of reboxetine, AXS-12, is under development for the treatment of EDS and cataplexy in narcolepsy. AXS-12 has demonstrated efficacy in reducing sleep and cataplexy in mice ${ }^{79}$ and decreasing EDS and cataplexy in a pilot study in individuals with narcolepsy. ${ }^{80} \mathrm{~A}$ recently completed phase 2 randomized, double-blind, placebo-controlled, crossover trial in individuals with narcolepsy and cataplexy compared AXS-12 with placebo and measured EDS, cataplexy, cognitive function, and sleep quality. ${ }^{81}$ Phase 3 trials are planned, and if AXS-12 proves efficacious in the treatment of both EDS and cataplexy, it may be an alternative for individuals who do not tolerate or respond to other regimens. AXS-12 may also be useful to individuals with NT1 and comorbid depression. $^{58}$

\section{Non-Pharmacologic Treatments: Lifestyle Changes and Psychological Therapies}

Lifestyle changes for treating narcolepsy symptoms are prescribed routinely to complement pharmacologic treatments. These most often include implementing scheduled naps and maintaining a regular sleep routine. ${ }^{82}$ Many studies demonstrate the positive impact of naps on alertness in people with narcolepsy. ${ }^{83}$ Other lifestyle alterations may be recommended including modifying work schedules based on individual needs, avoiding sleep-inducing situations and identifying sources of psychosocial support. ${ }^{83,84}$ A study of 42 people with narcolepsy (NT1: $\mathrm{n}=32$, NT2: $\mathrm{n}=10$ ) showed decreased cardiopulmonary fitness compared to age- and sex-matched controls and higher levels of sleepiness and more frequent cataplexy episodes were associated with lower levels of exercise tolerance. ${ }^{85}$ Although causality cannot be determined from this cross-sectional study, these finding raise the question of whether regular exercise may help improve narcolepsy symptoms in patients who are able to safely implement an exercise routine.

Narcolepsy symptoms have been shown to have significant impact on not only the physical but also the mental and social health of persons with narcolepsy. ${ }^{86,88}$ Thus, counseling and psychological therapies have also been suggested as potential treatment approaches to address both the symptoms of narcolepsy, as well as the associated psychological impact that can accompany the diagnosis. ${ }^{83,84,89}$ Although there is a shortage of controlled studies examining the benefits of non-pharmacological and lifestyle adjustments to treat narcolepsy, reports indicate that a large proportion of individuals rely on alternative treatment modalities to supplement medication. ${ }^{83}$ Medication alone is often insufficient for alleviating narcolepsy symptoms and psychological and lifestyle approaches should be considered more broadly and tested rigorously. ${ }^{42}$

\section{Challenges and Prospects for Specific Populations with Narcolepsy Children and Adolescents with Narcolepsy}

Narcolepsy may be diagnosed in children as young as 5 or 6 years of age, with a peak incidence around 15 years of age. ${ }^{90}$ Most children with narcolepsy are brought to their physicians' attention because of their excessive daytime sleepiness, similar to adults. ${ }^{91}$ Some children may restart taking naps after having given them up. The naps may not be refreshing, which may differ from adults, who often characterize their naps as refreshing. Some older children are able to realize they have cataplexy or muscle weakness with strong emotions, whereas others only realize what has been happening when discussing this with their physician. Parents may note the muscle weakness, yet not realize it to be cataplexy, such as when a child's head and tongue protrudes or thrusts forward while laughing which may also include ptosis (occulobuccofacial weakness), or may wonder why their child keeps falling to the floor when laughing. ${ }^{92}$ Children are sometimes so frightened by the hypnagogic and hypnopompic hallucinations that they may not volunteer that information unless specifically asked. Similarly, sleep paralysis can be difficult to verbalize for children. Sleep logs and video recordings showing cataplexy are helpful if the child and parent have difficulty articulating the pentad signs of narcolepsy.

Diagnosis is by the same "adult" criteria with in-lab polysomnography and multiple sleep latency testing, using 
the same cut-offs for the mean sleep latency of $</=8$ minutes and 2 sleep-onset REM periods (or sleep onset REM latency of $</=15$ minutes on overnight polysomnography) as described in detail earlier. Hypocretin levels can also be measured in children.

Treatment is also similar to adults with age-specific considerations. ${ }^{93}$ Lifestyle treatment is as important as medication. Children are advised to continue to maintain a good sleep-wake routine (consistent bedtimes and wake times) and live an active lifestyle, full of the usual extracurricular activities. Sports activities keep the child moving and improve alertness and energy levels. Parents are counseled to be especially vigilant regarding safety while using playground equipment, swimming, cooking, or any other activities during which if cataplexy occurred, the child could sustain significant harm. It is recommended that children with narcolepsy are always supervised during these types of 'potentially dangerous' activities. For teens who are old enough to drive, the young drivers are educated on the importance of first assessing if they are alert enough, driving only when necessary, driving short distances if possible, and driving in the right lane in case they need to pull over abruptly.

Parents are educated on the importance of healthy eating as some children may have substantial weight gain at the onset of their narcolepsy. Additionally, parents are instructed to look for signs of precocious puberty, which is associated with narcolepsy, and may need to be evaluated by an endocrinologist. ${ }^{94}$

Children and teens with narcolepsy are at higher risk for depression, social and emotional distress, aggressive behavior, and difficulty with focus and attention resulting in poor school performance. ${ }^{87,95,96}$ Treatment can substantially improve quality of life.

Most teachers are able to make accommodations for children to have one or more brief scheduled naps within the school day if needed, and otherwise, it is advised that children take a brief nap after school. Job counseling is also provided to teens with narcolepsy (with or without cataplexy) to avoid professions that require quiet, sedentary work, night-shift work, a commercial driver's license, or other jobs including but not limited to pilot, military personnel, security personnel. Additionally, for teens with cataplexy, counseling is provided to avoid jobs that include heavy machinery or heights.

Despite lifestyle changes, medications are almost always necessary to help children with narcolepsy stay awake and alert during the day. Parents may be hesitant to start medications; however, it is of paramount importance for the child to be alert, focused and able to keep up with their schoolwork. At the time of publication, there are no evidence-based guidelines or consensus guidelines for treatment of narcolepsy in children. Therapy is individualized for each child's symptoms. Typically, stimulants are first-line medications, followed by wake-promoting agents, including modafinil or armodafinil, and lastly, sodium oxybate. Cataplexy may be treated with sodium oxybate and antidepressant medications in the SSRI or SNRI classes. Sodium oxybate is now approved to treat EDS and cataplexy in children and adolescents age 7 years and older, ${ }^{48}$ the wake-promoting medications modafinil and armodafinil are approved for children 17 years of age and older, and pitolisant is being tested in pediatric populations. ${ }^{97}$ It is also important to discuss with older teens to avoid alcohol, which can worsen sleep quality and also possibly interact with medications.

\section{Individuals of Childbearing Potential}

The limited amount of information regarding narcolepsy during pregnancy and lactation and potential effects of common narcolepsy medications on the human fetus and neonate present challenges both to women with narcolepsy when facing decisions concerning family planning as well as to physicians treating this population of narcolepsy patients. A survey-based study evaluating narcolepsy specialists around the world indicated that most physicians stopped medications at the time of conception, during pregnancy and during breastfeeding. ${ }^{98}$ This report suggested that based on the available literature, perceived risks of narcolepsy medications might be overestimated, ${ }^{98}$ however current studies indicate the potential of teratogenicity of certain therapies and treatment of narcolepsy during pregnancy should consider all potential risks and benefits and should include informed collaboration between patient, sleep doctor and obstetrician. ${ }^{99,100}$ Challenges for women with narcolepsy who choose to go without medication during pregnancy and lactation are likely to include compromised safety to both mother and fetus/infant as well as increased work absences and potential for unemployment for working women due to poor symptom control. ${ }^{101}$ Simply anticipating these challenges could make embarking on family life a difficult consideration for young adult women with narcolepsy and may, in part, explain the increased age at first pregnancy for NT1 women and increased likelihood for single-pregnancy discovered in recent retrospective cohort and case-control studies. ${ }^{102,103}$ 
A recent finding of particular relevance to this population of people with narcolepsy is the demonstration of emission of sodium oxybate, first-line therapy for NT1, in the milk of lactating women taking sodium oxybate. ${ }^{104,105}$ Our study in 2 women with NT1 representing 3 pregnancies, and a case report in a woman with NT1 indicated the possibility of avoiding infant exposure to elevated levels of gamma-hydroxybutyric acid (GHB) by delaying feeding expressed milk until exogenous GHB can be presumed to be absent from the milk. Larger, confirmatory studies should be undertaken; however, this information should be considered when evaluating therapeutic options for NT1 women who elect to breastfeed and respond well to sodium oxybate therapy. This option may be less feasible for women who have limited support for night-time infant care and feedings. It is significant that in the retrospective cohort study by Maurovich-Horvat et al, ${ }^{102} 60.1 \%$ of women who developed narcolepsy before or during pregnancy reported neonatal care to be adversely affected by narcolepsy symptoms. Reported struggles in the puerperium included excessive sleepiness, sleep attacks during feeding or nursing, fear of being impaired by symptoms, and cataplexy while holding the baby. Improved options for safely treating postpartum NT1 women with sodium oxybate might help to eschew these dangers.

Considering the burden the above challenges could pose for the duration of pregnancy and breastfeeding in childbearing women with narcolepsy, it is requisite that this population be presented with a comprehensive understanding of their options, and sufficient guidance from their physician throughout the family planning process.

Studies examining the impact of narcolepsy on intimate relationships and sexuality are limited, but shed light on unique challenges to which practitioners should demonstrate sensitivity. Women and men with narcolepsy have reported forms of sexual dysfunction, including cataplexy with orgasm (termed orgasmolepsy) and erectile dysfunction with narcolepsy treatment, suggesting that individuals with narcolepsy may experience distinct challenges associated with sexual intimacy. ${ }^{106,109}$ Orgasmolepsy episodes have been described as involving complete loss of muscle tone, but not lasting more than 30 seconds. Case studies indicate that cataplexy treatments may be effective in reducing orgasmolepsy for some patients, but rigorous studies in this area have not been pursued. ${ }^{107} \mathrm{~A}$ further consideration, which receives little mention in the literature, is the potential impact of other symptoms of narcolepsy and narcolepsy treatments on intimate relationships and sexuality. ${ }^{110}$ Excessive sleepiness, strict sleep regimens and sedating properties or sexual dysfunction side effects of narcolepsy therapies are only a few of the aspects of disease presentation and management that could pose challenges to sexual intimacy and intimate partner relationships. Future research in this area could aid to increase sleep specialist-awareness of some underaddressed psychosocial challenges in narcolepsy.

\section{Acknowledging and Addressing Narcolepsy Stigma}

Public understanding of narcolepsy is limited and often inaccurate, with entertainment and media portrayals associating narcolepsy with humorously falling asleep rather than with a serious health condition requiring medical attention. ${ }^{11}$ As a result, people with narcolepsy face significant delays in proper detection and diagnosis. ${ }^{22}$ Misperceptions of narcolepsy also perpetuate stigma and being labeled by teachers, co-workers, supervisors, and others as antisocial, lazy, careless, or faking. ${ }^{42}$

Significant prior research over the past 38 years and across various cultures well established that narcolepsy significantly impacts health-related quality of life (HRQOL) and psychosocial wellbeing, e.g. ${ }^{88,112,119}$ People with narcolepsy report feeling socially isolated (even within their own families), inferior to others, and hesitant to disclose their disorder to others, fearing the consequences and reaction they would receive. ${ }^{112}$ Despite these descriptions of diminished quality-of-life, health-related stigma was not studied as a potential underlying mechanism impacting people with narcolepsy until $2015 .^{120}$ Defined as a social process, experienced or anticipated, characterized by exclusion, rejection, blame or devaluation that results from experience, perception or reasonable anticipation of an adverse social judgment about a person or a group, ${ }^{121}$ health-related stigma has been reported in a number of chronic conditions, and is a potential predictor of lower HRQOL. Kapella et al (2015) found that young adults with narcolepsy reported feeling significantly more stigma than those without narcolepsy in all domains, including social rejection, financial insecurity, internalized shame, and social isolation and that the levels of health-related stigma observed in this sample were comparable to those found in people living with HIV. ${ }^{120}$ These high levels of health-related stigma are significant, especially with growing evidence that stigma contributes to economic disparities and difficulties with social relationships, and can affect 
access to and the quality of health care as well as adherence to a medication regimen. ${ }^{122}$ In addition to high levels of perceived stigma, people with narcolepsy reported lower levels of quality of life, especially in physical, vitality and social functioning roles, and greater anxiety and depression than young adults in the comparison group. The major factors impacting social functioning were depression, sleepiness, and social rejection. Conversely, less depression, sleepiness and perceived social isolation predicted significantly better social functioning. Further, the authors proposed that health-related stigma likely affects social functioning both directly and indirectly through depressed mood.

\section{Addressing Stigma at an Individual Level}

Further research is needed to examine other levels of stigma (societal, organizational, and individual perspectives) and across a broader age range. From this, evidence-based strategies could be developed for predicting vulnerable individuals and implementing effective strategies to mitigate the deleterious impacts of stigma. Health-related stigma interventions in other chronic illness communities suggest benefits from using educational programs, skill-building, cognitive behavioral therapy and support groups.

Kapella et al ground-breaking first study on narcolepsy and stigma highlights that living with this condition is a multifaceted experience involving a complex web of social dynamics that extends beyond managing the basic symptoms of narcolepsy. ${ }^{120}$ Thus, treating the full scope of narcolepsy's impact requires a multidisciplinary care approach including pharmacologic treatments, scheduled naps, lifestyle changes (such as diet, exercise, and sleep hygiene) and social support (such as counseling, support groups, conferences, and educational and skill-building programs).

Connecting people with narcolepsy and their loved ones to patient advocacy organizations is another key way to support the social experiences of those living with narcolepsy. Patient advocacy organizations offer a variety of resources, events, support services and educational programming that can help to foster connection and reduce stigma. For example, sharing one's illness story with others is a powerful method of creating reflection and connection, and finding meaning and understanding. ${ }^{123}$ Particularly for "invisible illnesses" like narcolepsy, having one's voice heard can be an incredibly validating experience. ${ }^{124}$ Thus, there is great value in programs like Wake Up Narcolepsy's Narcolepsy 360 Podcast $^{125}$ and Narcolepsy Network's
Annual Conference. ${ }^{126}$ In addition to formal in-person and video support groups run through these organizations, there are additional formal and informal online support groups via platforms like Facebook and Patients Like Me.

\section{Addressing Stigma at a Societal Level}

During the FDA's narcolepsy meeting in 2013, people with narcolepsy and their loved ones consistently expressed the need for more to be done to help narcolepsy gain acceptance and understanding in schools, workplaces, and other social and professional settings. ${ }^{42}$ Thus, efforts aiming to raise accurate awareness and debunk societal misperceptions of narcolepsy will help to reduce stigma and reduce unnecessary delays to diagnosis.

Better public education may also reduce the restrictions that people with narcolepsy face in professional and educational settings. For example, a diagnosis of narcolepsy is listed as a disqualifying condition for serving in the US military and those with narcolepsy must apply for a medical waiver to enlist or continue to serve. ${ }^{127}$ Furthermore, sleep disorders, including narcolepsy, are associated with more auto accidents and medical errors in healthcare workers. ${ }^{128}$ In the United States, legal protections under the Americans with Disabilities Act of 1990 (ADA) can help people with narcolepsy to receive educational and employment accommodations. Determining whether a particular person with narcolepsy qualifies for educational or employment accommodations should be an individualized process that takes into consideration the person's particular circumstances. Likewise, while educational and employment accommodations both involve the ADA and use some overlapping terminology - different factors, processes and protections are in play in these two areas. Learning about the legal protections empowers people with narcolepsy and their doctors and caregivers to help those with narcolepsy to obtain appropriate accommodations and succeed at school and in the workplace.

Research indicates that audiences are more likely to engage with and retain information when it is delivered by someone with lived experience, giving a "face" to medical terminology and statistics. ${ }^{129}$ For example, In Our Own Voices (IOOV) is a public education program developed by the National Alliance on Mental Illness (NAMI) that trains individuals living with mental illness to deliver public presentations of their personal stories of illness and recovery. Studies have found that presentations by IOOV trainees are effective in increasing knowledge and 
correcting misperceptions among the general public, college students, and healthcare professionals alike. ${ }^{130,132}$

Following these best practices, Project Sleep created the Rising Voices of Narcolepsy $\operatorname{program}^{133}$ in 2017 to train people with narcolepsy to share their story effectively via speaking or writing. In just three years, the program has trained over 60 speakers and writers around the world. This program aims to empower advocates while also raising awareness, reducing stigma and reducing delays to diagnosis. Other patient advocacy organizations in the US include:

Hypersomnia Foundation:

https://www.hypersomniafoundation.org/

Narcolepsy Network:

https://narcolepsynetwork.org/

Project Sleep:

https://project-sleep.com

Wake Up Narcolepsy:

https://www.wakeupnarcolepsy.org/

\section{Conclusion}

Although narcolepsy is not a prevalent condition, it is one of the most common causes of excessive daytime sleepiness. ${ }^{2}$ Nevertheless, it is under-recognized by the general public and medical providers and people with narcolepsy often experience protracted delays in diagnosis. Testing for narcolepsy has relied on behavioral testing and clinical history but advances in biomarkers such as CSF hypocretin-1 levels and neuroimaging may make narcolepsy easier to diagnose and shed light on pathophysiologic differences between NT1 and NT2. Until recently, few pharmacologic treatment options were available, and most medications used to treat narcolepsy were not developed for this disorder and had not undergone vigorous testing in this population. New pharmaceuticals that are now available and those in development provide novel options to treat EDS and cataplexy. In the last decade, the experiences of people with narcolepsy are playing a more prominent role in management of this condition. Innovations in pharmacologic therapies, lifestyle modifications and behavioral strategies, and personalized care for specific populations are changing the outlook for management of narcolepsy. Finally, the acknowledgement of health-related stigma associated with narcolepsy and efforts to reduce the impact of this bias are allowing individuals with narcolepsy to enjoy a better quality of life.

\section{Disclosure}

Dr. Emily C. Barker is a medical writer and has worked as an independent contractor generating reports and summaries for medical advisory board meetings and round-table discussions for Jazz Pharmaceuticals and has also received speaker honoraria from Jazz Pharmaceuticals. Julie Flygare, JD is employed as the President \& CEO of Project Sleep, a 501(c)(3) non-profit organization dedicated to raising awareness about sleep health and sleep disorders. As a patient-perspective expert, Ms. Flygare has consulted on patient-centricity and health communications best practices with drug developers including Harmony Biosciences, Jazz Pharmaceuticals, Avadel Pharmaceuticals and Takeda Pharmaceuticals. She also receives royalties from sales of her book, Wide Awake \& Dreaming: A Memoir of Narcolepsy. Dr. Shalini Paruthi is a clinical educator and sleep medicine physician, with focus in pediatrics. She gives professional lectures and receives honoraria from conference organizers and royalties from UpToDate. Dr. Paruthi is an uncompensated member of the Board of Directors of the Restless Legs Syndrome Foundation. Dr. Katherine M. Sharkey is a sleep medicine physician; she recently participated in an early-access patient program for pitolisant sponsored by Harmony Biosciences. As an academic physician, she gives professional talks and receives honoraria from conference organizers and royalties from UpToDate. Her research is funded through grant support from NIH and the Hassenfeld Institute. Dr. Sharkey is an uncompensated founding member of TIMES UP Healthcare. The authors report no other conflicts of interest in this work.

\section{References}

1. AASM. The International Classification of Sleep Disorders - Third Edition (ICSD-3). 3rd ed. Vol. 3. Darien, IL: American Academy of Sleep Medicine; 2014.

2. Scammell TE. Narcolepsy. N Engl J Med. 2015;373(27):2654-2662. doi:10.1056/NEJMra1500587

3. Harris SF, Monderer RS, Thorpy M. Hypersomnias of central origin. Neurol Clin. 2012;30(4):1027-1044. doi:10.1016/j.ncl.2012.08.002

4. American Academy of Sleep Medicine. International Classification of Sleep Disorders. 3rd ed. Darien, IL: American Academy of Sleep Medicine; 2014.

5. Nishino S, Ripley B, Overeem S, Lammers GJ, Mignot E. Hypocretin (orexin) deficiency in human narcolepsy. Lancet (London, England). 2000;355(9197):39-40. doi:10.1016/S0140-6736(99)05582-8

6. Mayo Clinic Laboratories. Orexin-A/Hypocretin-1 in Spinal Fluid. available from: https://www.mayocliniclabs.com/test-catalog/Clinical +and+Interpretive/604230. Accessed April 2, 2020.

7. Thannickal TC, Nienhuis R, Siegel JM. Localized loss of hypocretin (orexin) cells in narcolepsy without cataplexy. Sleep. 2009;32 (8):993-998. doi:10.1093/sleep/32.8.993 
8. Baumann CR, Mignot E, Lammers GJ, et al. Challenges in diagnosing narcolepsy without cataplexy: a consensus statement. Sleep. 2014;37(6):1035-1042. doi:10.5665/sleep.3756

9. Pérez-Carbonell L, Leschziner G. Clinical update on central hypersomnias. J Thorac Dis. 2018;10(S1):S112-S123. doi:10.21 037/jtd.2017.10.161

10. Trotti LM. Idiopathic hypersomnia. Sleep Med Clin. 2017;12 (3):331-344. doi:10.1016/j.jsmc.2017.03.009

11. Mignot E, Hayduk R, Black J, Grumet FC, Guilleminault C. HLA DQB1*0602 is associated with cataplexy in 509 narcoleptic patients. Sleep. 1997;20(11):1012-1020.

12. Sarkanen TO, Alakuijala APE, Dauvilliers YA, Partinen MM. Incidence of narcolepsy after H1N1 influenza and vaccinations: systematic review and meta-analysis. Sleep Med Rev. 2018;38:177-186. doi:10.1016/j.smrv.2017.06.006

13. Longstreth WTJ, Koepsell TD, Ton TG, Hendrickson AF, van Belle G. The epidemiology of narcolepsy. Sleep. 2007;30 (1):13-26. doi:10.1093/sleep/30.1.13

14. Tashiro T, Kanbayashi T, Iijima S, Hishikawa Y. An epidemiological study of narcolepsy in Japanese (abstract). J Sleep Res. 1992;1(Suppl 1):228.

15. Kawai M, O’Hara R, Einen M, Lin L, Mignot E. Narcolepsy in African Americans. Sleep. 2015;38(11):1673-1681. doi:10.5665/ sleep. 5140

16. Lavie P, Peled R. Narcolepsy is a rare disease in Israel. Sleep. 1987;10(6):608-609. doi:10.1093/sleep/10.6.608

17. Scheer D, Schwartz SW, Parr M, Zgibor J, Sanchez-Anguiano A, Rajaram L. Prevalence and incidence of narcolepsy in a US health care claims database, 2008-2010. Sleep. 2019;42(7):7. doi:10.10 93/sleep/zsz091

18. Mallampalli MP, Carter CL. Exploring sex and gender differences in sleep health: a Society for Women's Health Research Report J Womens Health (Larchmt). 2014;23(7):553-562. doi:10.1089/ jwh.2014.4816

19. Cohen A, Mandrekar J, St Louis EK, Silber MH, Kotagal S. Comorbidities in a community sample of narcolepsy. Sleep Med. 2018;43:14-18. doi:10.1016/j.sleep.2017.11.1125

20. Won C, Mahmoudi M, Qin L, Purvis T, Mathur A, Mohsenin V. The impact of gender on timeliness of narcolepsy diagnosis. J Clin Sleep Med JCSM off Publ Am Acad Sleep Med. 2014;10 (1):89-95. doi: $10.5664 /$ jcsm. 3370

21. Riehl J, Nishino S, Cederberg R, Dement WC, Mignot E. Development of cataplexy in genetically narcoleptic Dobermans. Exp Neurol. 1998;152(2):292-302. doi:10.1006/exnr.1998.6847

22. Thorpy MJ, Krieger AC. Delayed diagnosis of narcolepsy: characterization and impact. Sleep Med. 2014;15(5):502-507. doi:10.1016/j.sleep.2014.01.015

23. Taddei RN, Werth E, Poryazova R, Baumann CR, Valko PO. Diagnostic delay in narcolepsy type 1: combining the patients' and the doctors' perspectives. J Sleep Res. 2016;25(6):709-715. doi: $10.1111 /$ jsr. 12420

24. Chervin RD, Aldrich MS. Sleep onset REM periods during multiple sleep latency tests in patients evaluated for sleep apnea. Am J Respir Crit Care Med. 2000;161(2 Pt 1):426-431. doi:10.1164/ ajrccm.161.2.9905071

25. Marti I, Valko PO, Khatami R, Bassetti CL, Baumann CR. Multiple sleep latency measures in narcolepsy and behaviourally induced insufficient sleep syndrome. Sleep Med. 2009;10 (10):1146-1150. doi:10.1016/j.sleep.2009.03.008

26. Lopez R, Doukkali A, Barateau L, et al. Test-Retest reliability of the multiple sleep latency test in central disorders of hypersomnolence. Sleep. 2017;40(12):12. doi:10.1093/sleep/zsx164

27. Andlauer O, Moore H, Jouhier L, et al. Nocturnal rapid eye movement sleep latency for identifying patients with narcolepsy/ hypocretin deficiency. JAMA Neurol. 2013;70(7):891-902. doi:10.1001/jamaneurol.2013.1589
28. Cairns A, Bogan R. Underutilization of the MSLT in sleepy patients with a short onset REM period (SOREMP) in the sleep clinic. Sleep Med. 2017;32:150-156. doi:10.1016/j.sleep.2016. 11.023

29. Cairns A, Bogan R. Prevalence and clinical correlates of a Short Onset REM Period (SOREMP) during Routine PSG. Sleep. 2015;38(10):1575-1581. doi:10.5665/sleep.5050

30. Mignot E, Lammers GJ, Ripley B, et al. The role of cerebrospinal fluid hypocretin measurement in the diagnosis of narcolepsy and other hypersomnias. Arch Neurol. 2002;59(10):1553-1562. doi:10.1001/archneur.59.10.1553

31. Cavaliere C, Longarzo M, Fogel S, Engstrom M, Soddu A. Neuroimaging of narcolepsy and primary hypersomnias. Neuroscientist. 2020;107385842090582. doi:10.1177/107385842 0905829

32. Black J, Reaven NL, Funk SE, et al. Medical comorbidity in narcolepsy: findings from the Burden of Narcolepsy Disease (BOND) study. Sleep Med. 2017;33:13-18. doi:10.1016/j. sleep.2016.04.004

33. Furuta H, Thorpy MJ, Temple HM. Comparison in symptoms between aged and younger patients with narcolepsy. Psychiatry Clin Neurosci. 2001;55(3):241-242. doi:10.1046/j.1440-1819.20 01.00841.x

34. Dauvilliers Y, Gosselin A, Paquet J, Touchon J, Billiard M, Montplaisir J. Effect of age on MSLT results in patients with narcolepsy-cataplexy. Neurology. 2004;62(1):46-50. doi:10.1212/ 01.WNL.0000101725.34089.1E

35. Büchele F, Baumann CR, Poryazova R, Werth E, Valko PO. Remitting narcolepsy? Longitudinal observations in a hypocretin-deficient cohort. Sleep. 2018;41(9). doi:10.1093/ sleep/zsy118

36. Almeneessier AS, Alballa NS, Alsalman BH, Aleissi S, Olaish AH, BaHammam AS. A 10-year longitudinal observational study of cataplexy in a cohort of narcolepsy Type 1 patients. Nat Sci Sleep. 2019;11:231-239. doi:10.2147/NSS. S229105

37. Chang E-T, Lin C-L, Chen S-F, Shen Y-C. Risk of burn injury in patients with narcolepsy: a nationwide population-based cohort study. J Burn Care Res. 2020;41(3):663-667. doi:10.1093/jbcr/ iraa004

38. Chang E-T, Lin C-L, Chen S-F, Hsu C-Y, Shen Y-C. Risk of bone fractures in patients with narcolepsy: a nationwide population-based cohort study. Sleep Med. 2020;70:55-59. doi:10.1016/j.sleep.2020.02.015

39. Pizza F, Jaussent I, Lopez R, et al. Car crashes and central disorders of hypersomnolence: a French study. PLoS One. 2015;10(6):e0129386. doi:10.1371/journal.pone.0129386

40. Driver and Vehicle Licensing Agency. Guidance, Neurological Disorders: assessing Fitness to Drive. Available from: https:// www.gov.uk/guidance/neurological-disorders-assessing-fitness-to -drive\#primarycentral-hypersomnias-including-narcolepsy. Accessed June 20, 2020. 2020.

41. Narcolepsy Network. Narcolepsy and driving laws. Available from: https://narcolepsynetwork.org/narcolepsydrivinglaws/. Accessed June 20, 2020.

42. United States Food and Drug Administration. The Voice of the Patient Report. Available from: https://www.fda.gov/media/ 88736/download. 2014. Accessed April 3, 2020.

43. Maski K, Steinhart E, Williams D, et al. Listening to the patient voice in narcolepsy: diagnostic delay, disease burden, and treatment efficacy. J Clin Sleep Med JCSM off Publ Am Acad Sleep Med. 2017;13(3):419-425. doi:10.5664/ jesm.6494

44. Perez-Carbonell L, Lyons E, Gnoni V, et al. Adherence to wakefulness promoting medication in patients with narcolepsy. Sleep Med. 2020;70:50-54. doi:10.1016/j.sleep.2020.02.013 
45. Black SW, Yamanaka A, Kilduff TS. Challenges in the development of therapeutics for narcolepsy. Prog Neurobiol. 2017;152:89-113. doi:10.1016/j.pneurobio.2015.12.002

46. Swick TJ. Treatment paradigms for cataplexy in narcolepsy: past, present, and future. Nat Sci Sleep. 2015;7:159-169. doi:10.2147/ NSS.S92140

47. Barateau L, Dauvilliers Y. Recent advances in treatment for narcolepsy. Ther Adv Neurol Disord. 2019;12:1756286419 875622. doi:10.1177/1756286419875622

48. Plazzi G, Ruoff C, Lecendreux M, et al. Treatment of paediatric narcolepsy with sodium oxybate: a double-blind, placebo-controlled, randomised-withdrawal multicentre study and open-label investigation. Lancet Child Adolesc Heal. 2018;2 (7):483-494. doi:10.1016/S2352-4642(18)30133-0

49. Golicki D, Bala MM, Niewada M, Wierzbicka A. Modafinil for narcolepsy: systematic review and meta-analysis. Med Sci Monit Int Med J Exp Clin Res. 2010;16(8):RA177-86.

50. Bogan RK, Feldman N, Emsellem HA, et al. Effect of oral JZP-110 (ADX-N05) treatment on wakefulness and sleepiness in adults with narcolepsy. Sleep Med. 2015;16(9):1102-1108. doi:10.1016/j.sleep.2015.05.013

51. Ruoff C, Swick TJ, Doekel R, et al. Effect of oral JZP-110 (ADX-N05) on wakefulness and sleepiness in adults with narcolepsy: a Phase 2b study. Sleep. 2016;39(7):1379-1387. doi:10.56 65/sleep.5968

52. Thorpy MJ, Shapiro C, Mayer G, et al. A randomized study of solriamfetol for excessive sleepiness in narcolepsy. Ann Neurol. 2019;85(3):359-370. doi:10.1002/ana.25423

53. Schweitzer PK, Rosenberg R, Zammit GK, et al. Solriamfetol for excessive sleepiness in obstructive sleep apnea (TONES 3). A randomized controlled trial. Am J Respir Crit Care Med. 2019;199(11):1421-1431. doi:10.1164/rccm.201806-1100OC

54. Wisor J. Modafinil as a catecholaminergic agent: empirical evidence and unanswered questions. Front Neurol. 2013;4:139. doi:10.3389/fneur.2013.00139

55. Rothman RB, Baumann MH, Dersch CM, et al. Amphetaminetype central nervous system stimulants release norepinephrine more potently than they release dopamine and serotonin. Synapse. 2001;39(1):32-41. doi:10.1002/1098-2396(20010101) 39:1<32::AID-SYN5 $>3.0 . \mathrm{CO} ; 2-3$

56. Baladi MG, Forster MJ, Gatch MB, et al. Characterization of the neurochemical and behavioral effects of solriamfetol (JZP-110), a selective dopamine and norepinephrine reuptake inhibitor. J Pharmacol Exp Ther. 2018;366(2):367-376. doi:10.1124/ jpet.118.248120

57. Ruoff C, Bogan RK, Emsellem H, et al. Evaluation of the effect of JZP-110 in patients with narcolepsy assessed using the maintenance of wakefulness test censored to 20 minutes. Sleep Med. 2017;35:12-16. doi:10.1016/j.sleep.2017.03.025

58. Thorpy MJ. Recently approved and upcoming treatments for narcolepsy. CNS Drugs. 2020;34(1):9-27. doi:10.1007/s40263019-00689-1

59. Cephalon Inc. Nuvigil [Package Insert]. Frazer, PA; 2010.

60. Syed YY. Pitolisant: first Global Approval. Drugs. 2016;76 (13):1313-1318. doi:10.1007/s40265-016-0620-1

61. Baronio D, Gonchoroski T, Castro K, Zanatta G, Gottfried C, Riesgo R. Histaminergic system in brain disorders: lessons from the translational approach and future perspectives. Ann Gen Psychiatry. 2014;13(1):34. doi:10.1186/s12991-014-0034-y

62. Parmentier R, Anaclet C, Guhennec C, et al. The brain H3-receptor as a novel therapeutic target for vigilance and sleep-wake disorders. Biochem Pharmacol. 2007;73(8):11 57-1171. doi:10.1016/j.bcp.2007.01.002

63. Calik MW. Update on the treatment of narcolepsy: clinical efficacy of pitolisant. Nat Sci Sleep. 2017;9:127-133. doi:10.2147/ NSS.S103462
64. Dauvilliers Y, Bassetti C, Lammers GJ, et al. Pitolisant versus placebo or modafinil in patients with narcolepsy: a double-blind, randomised trial. Lancet Neurol. 2013;12(11):1068-1075. doi:10.1016/S1474-4422(13)70225-4

65. Inocente $\mathrm{C}$, Arnulf I, Bastuji $\mathrm{H}$, et al. Pitolisant, an inverse agonist of the histamine $\mathrm{H} 3$ receptor: an alternative stimulant for narcolepsy-cataplexy in teenagers with refractory sleepiness. Clin Neuropharmacol. 2012;35(2):55-60. doi:10.1097/WNF.0b01 3e318246879d

66. Szakacs Z, Dauvilliers Y, Mikhaylov V, et al. Safety and efficacy of pitolisant on cataplexy in patients with narcolepsy: a randomised, double-blind, placebo-controlled trial. Lancet Neurol. 2017;16 (3):200-207. doi:10.1016/S1474-4422(16)30333-7

67. Clinicaltrials.gov. Once-nightly sodium oxybate for treatment of excessive daytime sleepiness and cataplexy in narcolepsy NCT02720744. Available from: https://clinicaltrials.gov/ct2/ show/NCT02720744. Accessed April 3, 2020.

68. Jazz Pharmaceuticals. Prescribing Information for Xyrem ${ }^{\circledR}$ (Sodium Oxybate) Oral Solution; 2012.

69. Whelton PK, Appel LJ, Sacco RL, et al. Sodium, blood pressure, and cardiovascular disease: further evidence supporting the American Heart Association sodium reduction recommendations. Circulation. 2012;126(24):2880-2889. doi:10. 1161/CIR.0b013e318279acbf

70. Bogan R, Thorpy M, Dauvilliers Y, et al. Efficacy and safety of Jzp-258 in a Phase 3 double-blind, placebo-controlled, randomised-withdrawal study in adults with narcolepsy with cataplexy. Sleep Med. 2019;64:S43. doi:10.1016/j.sleep.2019. 11.120

71. Dauvilliers Y, Šonka K, Bogan RK, et al. Changes in cataplexy frequency by prior therapy in a phase 3 , double-blind, placebo-controlled, randomised withdrawal study of JZP-258 in adults with narcolepsy with cataplexy. Sleep Med. 2019;64:S86. doi:10.1016/j.sleep.2019.11.235

72. Nishino S, Okuro M, Kotorii N, et al. Hypocretin/orexin and narcolepsy: new basic and clinical insights. Acta Physiol (Oxf). 2010;198(3):209-222. doi:10.1111/j.1748-1716.2009.02012.x

73. Yukitake H, Fujimoto T, Ishikawa T, et al. TAK-925, an orexin 2 receptor-selective agonist, shows robust wake-promoting effects in mice. Pharmacol Biochem Behav. 2019;187:172794. doi:10.10 16/j.pbb.2019.172794

74. Evans R, Tanaka S, Tanaka S, et al. A Phase 1 single ascending dose study of a novel orexin 2 receptor agonist, TAK-925, in healthy volunteers (HV) and subjects with narcolepsy type 1 (NT1) to assess safety, tolerability, pharmacokinetics, and pharmacodynamic outcomes. Sleep Med. 2019;64:S105-S106. doi:10.1016/j.sleep.2019.11.290

75. Evans R, Hazel J, Faessel H, et al. Results of a phase 1, 4-period crossover, placebo-controlled, randomized, single dose study to evaluate the safety, tolerability, pharmacokinetics, and pharmacodynamics of TAK-925, a novel orexin 2 receptor agonist, in sleep-deprived healthy adults, utili. Sleep Med. 2019;64:S106. doi:10.1016/j.sleep.2019.11.291

76. Kimura H, Ishikawa T, Suzuki M. A novel, orally available orexin 2 receptor-selective agonist, tak-994, ameliorates narcolepsy-like symptoms in narcolepsy mouse models. Sleep Med. 2019;64: S199. doi:10.1016/j.sleep.2019.11.554

77. Ishikawa T, Suzuki M, Kajita Y, Miyanohana Y, Koike T, Kimura H. Discovery of a novel, orally available Orexin 2 receptor-selective agonist, TAK-994, as a therapeutic drug for narcolepsy. Sleep Med. 2019;64:S170. doi:10.1016/j.sleep.20 19.11.468

78. A Study of TAK-994 in Participants With Narcolepsy With or Without Cataplexy (Narcolepsy Type 1 [NT1] or Narcolepsy Type 2 [NT2]) - NCT04096560. Available from: Clinicaltrials. gov. Accessed June 20, 2020. 
79. Schmidt C, Leibiger J, Fendt M. The norepinephrine reuptake inhibitor reboxetine is more potent in treating murine narcoleptic episodes than the serotonin reuptake inhibitor escitalopram. Behav Brain Res. 2016;308:205-210. doi:10.1016/j.bbr.2016.04.033

80. Larrosa O, de la Llave Y, Bario S, Granizo JJ, Garcia-Borreguero D. Stimulant and anticataplectic effects of reboxetine in patients with narcolepsy: a pilot study. Sleep. 2001;24(3):282-285. doi:10.1093/sleep/24.3.282

81. Clinical Outcomes in Narcolepsy and Cataplexy: An Evaluation of Reboxetine Treatment (CONCERT) - NCT03881852. Available from: Clinicaltrials.gov. Accessed June 20, 2020.

82. Thorpy MJ, Dauvilliers Y. Clinical and practical considerations in the pharmacologic management of narcolepsy. Sleep Med. 2015;16(1):9-18. doi:10.1016/j.sleep.2014.10.002

83. Monderer R, Harris SF, Thorpy MJ. Non-pharmacologic treatments of narcolepsy. In: Narcolepsy. Springer; 2010:313-322.

84. Marin Agudelo HA, Jimenez Correa U, Carlos Sierra J, PandiPerumal SR, Schenck CH. Cognitive behavioral treatment for narcolepsy: can it complement pharmacotherapy? Sleep Sci (Sao Paulo, Brazil). 2014;7(1):30-42. doi:10.1016/j.slsci.2014.07.023

85. Matoulek M, Tuka V, Fialova M, Nevsimalova S, Sonka K. Cardiovascular fitness in narcolepsy is inversely related to sleepiness and the number of cataplexy episodes. Sleep Med. 2017;34:7-12. doi:10.1016/j.sleep.2017.02.017

86. Goswami M. The influence of clinical symptoms on quality of life in patients with narcolepsy. Neurology. 1998;50(2 Suppl 1):S316. doi:10.1212/wnl.50.2_suppl_1.s31

87. Avis KT, Shen J, Weaver P, Schwebel DC. Psychosocial characteristics of children with central disorders of hypersomnolence versus matched healthy children. J Clin Sleep Med JCSM off Publ Am Acad Sleep Med. 2015;11(11):1281-1288. doi:10.5664/jcsm.5186

88. Raggi A, Plazzi G, Ferri R. Health-related quality of life in patients with narcolepsy: a review of the literature. J Nerv Ment Dis. 2019;207(2):84-99. doi:10.1097/NMD.0000000000000918

89. Bhattarai J, Sumerall S. Current and future treatment options for narcolepsy: a review. Sleep Sci (Sao Paulo, Brazil). 2017;10 (1):19-27. doi:10.5935/1984-0063.20170004

90. Challamel MJ, Mazzola ME, Nevsimalova S, Cannard C, Louis J, Revol M. Narcolepsy in children. Sleep. 1994;17(8 Suppl):S1720. doi:10.1093/sleep/17.supp1_8.s17

91. Plazzi G, Clawges HM, Owens JA. Clinical characteristics and burden of illness in pediatric patients with narcolepsy. Pediatr Neurol. 2018;85:21-32. doi:10.1016/j.pediatrneurol.2018.06.008

92. Serra L, Montagna P, Mignot E, Lugaresi E, Plazzi G. Cataplexy features in childhood narcolepsy. Mov Disord. 2008;23 (6):858-865. doi:10.1212/01.WNL.0000101725.34089.1E

93. Kotagal S. Treatment of narcolepsy and other organic hypersomnias in children. Paediatr Respir Rev. 2018;25:19-24. doi:10.1016/j.prrv.2017.06.012

94. Maia Palhano AC, Kim LJ, Moreira GA, Santos Coelho FM, Tufik S, Levy Andersen M. Narcolepsy, precocious puberty and obesity in the pediatric population: a literature review. Pediatr Endocrinol Rev. 2018;16(2):266-274. doi:10.17458/per.vol16.20 18.Narcolepsypubertyobesity

95. Stores G, Montgomery P, Wiggs L. The psychosocial problems of children with narcolepsy and those with excessive daytime sleepiness of uncertain origin. Pediatrics. 2006;118(4):e1116-23. doi:10.1542/peds.2006-0647

96. Blackwell JE, Alammar HA, Weighall AR, Kellar I, Nash HM. A systematic review of cognitive function and psychosocial well-being in school-age children with narcolepsy. Sleep Med Rev. 2017;34:82-93. doi:10.1016/j.smrv.2016.07.003

97. Lecendreux M, Plazzi G, Franco P, et al. Pharmacokinetics of pitolisant in children and adolescents with narcolepsy. Sleep Med. 2020;66:220-226. doi:10.1016/j.sleep.2019.10.024
98. Thorpy M, Zhao CG, Dauvilliers Y. Management of narcolepsy during pregnancy. Sleep Med. 2013;14(4):367-376. doi:10.1016/j. sleep.2012.11.021

99. McLafferty LP, Spada M, Gopalan P. Pharmacologic treatment of sleep disorders in pregnancy. Sleep Med Clin. 2018;13 (2):243-250. doi:10.1016/j.jsmc.2018.02.004

100. Miller MA, Mehta N, Clark-Bilodeau C, Bourjeily G. Sleep pharmacotherapy for common sleep disorders in pregnancy and lactation. Chest. 2020;157(1):184-197. doi:10.1016/j.chest.2019.09.026

101. Kay-Stacey M, Attarian HP. Managing sleep disorders during pregnancy. Gend Genome. 2017;1(1):34-45. doi:10.1089/gg.20 16.0006

102. Maurovich-Horvat E, Kemlink D, Hogl B, et al. Narcolepsy and pregnancy: a retrospective European evaluation of 249 pregnancies. J Sleep Res. 2013;22(5):496-512. doi:10.1111/jsr.12047

103. Calvo-Ferrandiz E, Peraita-Adrados R. Narcolepsy with cataplexy and pregnancy: a case-control study. J Sleep Res. 2018;27 (2):268-272. doi:10.1111/jsr.12567

104. Busardo FP, Bertol E, Mannocchi G, et al. Determination of GHB levels in breast milk and correlation with blood concentrations. Forensic Sci Int. 2016;265:172-181. doi:10.1016/j.forsciint.2016. 02.020

105. Barker EC, Puchowicz M, Letterio J, Higgins K, Sharkey KM GHB levels in breast milk of women with narcolepsy with cataplexy treated with sodium oxybate. Sleep Med. 2017;36:172-177. doi:10.1016/j.sleep.2017.05.008

106. Reinert AE, Simon JA. "Did you climax or are you just laughing at me?" Rare phenomena associated with orgasm. Sex Med Rev. 2017;5(3):275-281. doi:10.1016/j.sxmr.2017.03.004

107. Poryazova R, Khatami R, Werth E, Bassetti CL. Weak with sex: sexual intercourse as a trigger for cataplexy. J Sex Med. 2009;6 (8):2271-2277. doi:10.1111/j.1743-6109.2009.01328.x

108. Roy A. Anorgasmia and cataplexy. Arch Sex Behav. 1977;6 (5):437-439. doi:10.1007/BF01541186

109. Karacan I. Erectile Dysfunction in Narcoleptic Patients. Sleep. 1986;9(1):227-231. doi:10.1093/sleep/9.1.227

110. Lindsley G. Narcolepsy, intimacy, and sexuality In: Goswami M, Thorpy M, Pandi-Perumal S, editors. Narcolepsy: A Clinical Guide. 2nd ed. Heidelberg, Germany: Springer; 2016:217-231.

111. Flygare J, Parthasarathy S. Narcolepsy: let the patient's voice awaken us! $A m$ J Med. 2015;128(1):10-13. doi:10.1016/j. amjmed.2014.05.037

112. Broughton WA, Broughton RJ. Psychosocial impact of narcolepsy. Sleep. 1994;17(8 Suppl):S45-9. doi:10.1093/sleep/17.suppl_8.s45

113. Ervik S, Abdelnoor M, Heier MS, Ramberg M, Strand G. Healthrelated quality of life in narcolepsy. Acta Neurol Scand. 2006;114 (3):198-204. doi:10.1111/j.1600-0404.2006.00594.x

114. Daniels E, King MA, Smith IE, Shneerson JM. Health-related quality of life in narcolepsy. J Sleep Res. 2001;10(1):75-81. doi:10.1046/j.1365-2869.2001.00234.x.

115. Ong JC, Fox RS, Brower RF, Mazurek S, Moore C. How does narcolepsy impact health-related quality of life? A mixed-methods study. Behav Sleep Med. 2020;1-14. doi:10.1080/15402002.2020.1715411

116. Dodel R, Peter H, Spottke A, et al. Health-related quality of life in patients with narcolepsy. Sleep Med. 2007;8(7-8):733-741. doi:10.1016/j.sleep.2006.10.010

117. Ozaki A, Inoue Y, Nakajima T, et al. Health-related quality of life among drug-naive patients with narcolepsy with cataplexy, narcolepsy without cataplexy, and idiopathic hypersomnia without long sleep time. J Clin Sleep Med JCSM off Publ Am Acad Sleep Med. 2008;4(6):572-578.

118. Vignatelli L, Plazzi G, Peschechera F, Delaj L, D’Alessandro R. A 5-year prospective cohort study on health-related quality of life in patients with narcolepsy. Sleep Med. 2011;12(1):19-23. doi:10.1016/j.sleep.2010.07.008 
119. David A, Constantino F, Dos Santos JM, Paiva T. Health-related quality of life in Portuguese patients with narcolepsy. Sleep Med. 2012;13(3):273-277. doi:10.1016/j.sleep.2011.06.021

120. Kapella MC, Berger BE, Vern BA, Vispute S, Prasad B, Carley DW. Health-related stigma as a determinant of functioning in young adults with narcolepsy. PLoS One. 2015;10(4): e0122478. doi:10.1371/journal.pone.0122478

121. Weiss MG, Ramakrishna J, Somma D. Health-related stigma: rethinking concepts and interventions. Psychol Health Med. 2006;11(3):277-287. doi:10.1080/13548500600595053

122. Hatzenbuehler ML, Phelan JC, Link BG. Stigma as a fundamental cause of population health inequalities. Am J Public Health. 2013;103(5):813-821. doi:10.2105/AJPH.2012.301069

123. Ryan E. Finding a new voice: writing through health adversity. J Lang Soc Psychol. 2006;25(4):423-436. doi:10.1177/0261927X06292768

124. Sallinen M, Kukkurainen ML, Peltokallio L. Finally heard, believed and accepted-peer support in the narratives of women with fibromyalgia. Patient Educ Couns. 2011;85(2):e126-30. doi:10.1016/j.pec.2011.02.011

125. Wake Up Narcolepsy. Narcolepsy 360 Podcast. Available from: https://www.wakeupnarcolepsy.org/get-involved/podcast/. Accessed April 3, 2020.

126. Narcolepsy Network. Narcolepsy network annual conference. https://narcolepsyconference.org/. Accessed June 20, 2020. Accessed April 3, 2020.

127. Office of the Under Secretary of Defense for Personnel and Readiness. Available from: DOD Instruction 6130.03 Medical Standards for Appointment, Enlistment, or Induction into the Military Services.; 2018. https://www.esd.whs.mil/Portals/54/ Documents/DD/issuances/dodi/613003p.pdf?ver=2018-05-04113917-883. Accessed May 26, 2020..
128. Weaver MD, Vetter C, Rajaratnam SMW, et al. Sleep disorders, depression and anxiety are associated with adverse safety outcomes in healthcare workers: a prospective cohort study. J Sleep Res. 2018;27(6):e12722. doi:10.1111/jsr.12722

129. Corrigan PW, Rafacz JD, Hautamaki J, et al. Changing stigmatizing perceptions and recollections about mental illness: the effects of NAMI's in our own voice. Community Ment Health J. 2010;46 (5):517-522. doi:10.1007/s10597-009-9287-3

130. Brennan M, McGrew JH. Evaluating the effects of NAMI's consumer presentation program, in our own voice. Psychiatr Rehabil J. 2013;36(2):72-79. doi:10.1037/h0094974

131. Wood AL, Wahl OF. Evaluating the effectiveness of a consumer-provided mental health recovery education presentation. Psychiatr Rehabil J. 2006;30(1):46-53. doi:10.2975/30.2006.46.53

132. Pittman JOE, Noh S, Coleman D. Evaluating the effectiveness of a consumer delivered anti-stigma program: replication with graduate-level helping professionals. Psychiatr Rehabil J. 2010;33(3):236-238. doi:10.2975/33.3.2010.236.238

133. Project Sleep. Rising voices of narcolepsy. Available from: https://project-sleep.com/rising-voices-of-narcolepsy/. Accessed April 3, 2020.

\section{Publish your work in this journal}

Nature and Science of Sleep is an international, peer-reviewed, open access journal covering all aspects of sleep science and sleep medicine, including the neurophysiology and functions of sleep, the genetics of sleep, sleep and society, biological rhythms, dreaming, sleep disorders and therapy, and strategies to optimize healthy sleep.
The manuscript management system is completely online and includes a very quick and fair peer-review system, which is all easy to use. Visit http://www.dovepress.com/testimonials.php to read real quotes from published authors. 